\section{$\underset{\substack{\text { hommes } \\ \text { \& migrations }}}{ }$}

\section{Hommes \& migrations}

Revue française de référence sur les dynamiques

migratoires

1302 | 2013

Le Japon, pays d'immigration?

\title{
Esquisse d'une sociologie des banlieues au Japon
}

\section{Chikako Mori}

\section{OpenEdition \\ Journals}

\section{Édition électronique}

URL : http://journals.openedition.org/hommesmigrations/2458

DOI : 10.4000/hommesmigrations.2458

ISSN : 2262-3353

\section{Éditeur}

Musée national de l'histoire de l'immigration

\section{Édition imprimée}

Date de publication : 1 avril 2013

Pagination : 45-55

ISBN : 978-2-919040-22-3

ISSN : $1142-852 X$

\section{Référence électronique}

Chikako Mori, «Esquisse d'une sociologie des banlieues au Japon », Hommes \& migrations [En ligne], 1302 | 2013, mis en ligne le 31 décembre 2015, consulté le 02 mai 2019. URL : http://

journals.openedition.org/hommesmigrations/2458; DOI : 10.4000/hommesmigrations.2458 


\title{
ESQUISSE \\ D'UNE SOCIOLOGIE \\ DES BANLIEUES \\ AU JAPON
}

par CHIKAKO MORI, maître de conférences en sociologie, sociologue, université Hitotsubashi ${ }^{1}$

\author{
Depuis une vingtaine d'années, l'État nippon a amélioré les \\ conditions d'entrée des résidents étrangers dans le parc locatif \\ public. Conséquence : les populations étrangères ont tendance \\ à se concentrer dans les banlieues des villes japonaises à côté \\ d'autres populations fragiles. Cette distribution sociologique \\ n'est pas sans rappeler la situation française, où la relégation \\ spatiale se conjugue aux difficultés socio-économiques. \\ Ici, comme là-bas, le racisme n'est souvent que le paravent de \\ la misère sociale dont souffrent les habitants des cités.
}

\section{La situation des cités HLM à forte population étrangère}

À première vue, il n'y a rien de commun entre les banlieues françaises et japonaises, car ces dernières ont été conçues et développées sur la base d'un modèle anglo-saxon. Si la première banlieue chic de Tokyo a été construite dès 1918 par Eiichi Shibusawa, homme d'affaires et "père du capitalisme japonais", sur le modèle britannique de la "citéjardin" (il s'agit de Denen-Chôfu, située au sud de la capitale), le développement massif des banlieues japonaises à partir des années 1950 a suivi les grandes lignes du modèle américain.

Ce n'est d'ailleurs pas un hasard si de nombreux ouvrages consacrés aux banlieues japonaises ont été écrits par des chercheurs américanistes ${ }^{2}$, qui les décrivent invariablement comme un lieu de résidence pour les classes moyennes, parfois comme de simples "cités-dortoirs", parfois comme un espace valorisant où les gens réalisent leur rêve d'avoir un

1. Chikako Mori est docteur en sociologie (EHESS) et maître de conférences à l'université Hitotsubashi de Tokyo. Son travail porte sur la sociologie comparée des inégalités et des marginalités urbaines, du racisme et des discriminations, dans une perspective comparatiste entre le Japon, l’Europe et les États-Unis. Ce travail a été réalisé grâce au concours de la Japan Society for the Promotion of Science (Grant-in-Aid for Scientific Research C : 24530677) et au programme CHORUS “Initiatives locales contre l'exclusion des résidents étrangers" (ANR-JSPS). 2. Masaaki Oba, Suburbia no Yûtsu (Mélancolie des Suburbia), Tokyo, Tokyo Shoseki, 1993 ; Atsushi Miura, Kazoku to Kôgai no Shakaigaku (Sociologie de la famille et des banlieues), Tokyo, PHP, 1995 ; Akihiko Nishizawa, "Kôgai to iu Meikyû" ("Un labyrinthe nommé banlieue"), in Toshi no Shakaigaku (Sociologie de la ville), Tokyo, Yûikaku, 2000 ; Mikio Wakabayashi, Kôgai to Gendaishakai (Les Banlieues et la Société contemporaine), Tokyo, Seikyûsha, 2000 ; Mikio Wakabayashi, Kôgai no Shakaigaku (Sociologie de la banlieue), Tokyo, Chikuma Shobô, 2007. 
Logements collectifs situés à quelques mètres des rues principales de Shin-Okubo. Un quartier organisé autour de commerces coréens et chinois. @ Camille Millerand

"home, sweet home $e^{3 "}$. Pour paradoxal que cela puisse paraître, la banlieue japonaise a toutes les allures, en ses débuts, du "rêve américain".

Cependant, nous assistons depuis une décennie à un changement de paradigme, qui aboutit aujourd'hui à une image beaucoup moins positive : on parle désormais de plus en plus du vieillissement de la population (pour n'en citer qu'un exemple, dans une cité de 1404 logements située dans la banlieue sud de Tokyo, un habitant sur deux a plus de 65 ans $^{4}$ ), de l'inquiétante décroissance démographique ou des "shrinking cities". C'est ainsi qu'Ha- toyama, une ville nouvelle à une heure et demie en train du centre de Tokyo, a perdu la moitié de sa population entre 1995 et $2005^{6}$ ! Sont aussi régulièrement évoqués les problèmes de la jeunesse : perte de repères, actes violents, brimades, suicides ${ }^{7} .$. Malgré cette évolution, un élément n'est toutefois jamais remis en question, qui semble constituer un véritable impensé de la société japonaise. Cet impensé, c'est le mythe de l'homogénéité : pour l'opinion publique - forgée notamment par les médias - comme pour la grande majorité des spécialistes, la banlieue serait constituée de résidents nationaux issus des classes moyennes au sens large du terme ${ }^{8}$.

3. Takashi Nakazawa, “Geography reality of suburban housing”, in Kanto Toshigakkai Nenpô, 2005. 4. Chikako Mori, "Shisetuka suru Kôeidanshi" ("La transformation des cités de banlieue en centres sociaux"), in Gendai Shisô, vol.34-14, Tokyo, Seidosha, 2006. 5. Marcel Langner, Wilfried Endlicher (dir.), Shrinking cities : effects on urban ecology and challenges for urban development, New York, Peter Lang, 2008. 6. “Kôgaibyô' no Kyôfu” (“La peur de la 'maladie de la banlieue"”), in AERA, Tokyo, éd. Asahi, 16 novembre 2006. 7. Shinji Miyadai, Maboroshi no Kôgai (Les Banlieues de l'illusion - l'avenir des jeunes qui évoluent dans une société postmoderne), Tokyo, Asahi, 1997 ; Mitsuo Oda, "Kôgai” no Tanjô to Shi (Naissance et mort de la "banlieue"), Tokyo, Seikyûsha, 1997. 8. Hiroki Azuma, Akihiro Kitada, Tokyo kara kangaeru (Penser à partir de Tokyo. Inégalités, banlieues et nationalisme), Tokyo, NHK Books, 2007; Atsushi Miura, "Fastfood-ka” suru Nihon (La "Fastfoodisation" du Japon. La périurbanisation et ses pathologies), Tokyo, Yosensha, 2004. 
Il s'agit en quelque sorte d'une double homogénéité fantasmée : il n'y aurait pas dans les banlieues japonaises de figure de l'Autre (ni social, ni ethnique), comme s'il n'y résidait que des Japonais, ayant de surcroît une certaine stabilité sociale, professionnelle et financière.

Or, si l'on y regarde de plus près, la réalité est beaucoup plus complexe. N'existe-t-il pas aujourd'hui un tout autre visage de la banlieue japonaise, marquée non par l'homogénéité, mais au contraire par l'hétérogénéité et l'instabilité ? De ce point de vue, la référence aux banlieues françaises pourraitelle nous aider à penser "la" banlieue japonaise ? Pour répondre à ces questions, nous nous intéresserons à ce qu'on appelle au Japon les "cités à forte population étrangère", souvent situées dans les banlieues excentrées, et dont nous préciserons la définition. Nous tenterons de montrer pour quelles raisons et dans quelles proportions les populations étrangères se sont concentrées dans certaines cités au cours des vingt dernières années. Puis, nous essaierons d'analyser, à partir d'un travail de terrain réalisé dans trois cités situées dans les banlieues de Nagoya et de Tokyo, l'impact de l'évolution démographique au niveau local, et ce que cette situation révèle des mutations actuelles du paysage suburbain japonais qui doivent se comprendre en relation avec les migrations asiatiques et internationales. Il s'agit de montrer que les problèmes liés aux banlieues doivent être considérés comme un "fait social total", comme l'avait excellemment formulé Abdelmalek Sayad par rapport à l'immigration, en reprenant une expression de Marcel Mauss : parler de la banlieue, "'est parler de la société en son entier, dans sa dimension diachro- nique, c'est-à-dire dans une perspective historique (...), et aussi dans son extension synchronique, c'est-à-dire du point de vue des structures présentes de la société et de leur fonctionnement ${ }^{9 "}$.

\section{L'apparition des cités à forte population étrangère}

Un constat pour commencer : depuis quinze ans, le nombre de foyers étrangers dans le parc des logements publics ${ }^{10}$ a été multiplié par $4,6^{11}$.

Cette augmentation est principalement due à ce qu'on appelle les "new comers" (des étrangers arrivés depuis les années 1980, par contraste avec les "old comers", population issue de l'immigration coloniale), à savoir les Brésiliens, les Philippins ou une partie des Chinois et autres Asiatiques (Coréens du Sud, Vietnamiens, Cambodgiens, Indonésiens, etc.). Si on regarde les chiffres, le phénomène peut paraître beaucoup moins important qu'en
Cet impensé, c'est le mythe de l'homogénéité : pour l'opinion publique - forgée notamment par les médias comme pour la grande majorité des spécialistes, la banlieue serait constituée de résidents nationaux issus des classes moyennes au sens large du terme. des 2,95 millions de logements publics sont occupés par des foyers étrangers au Japon ${ }^{12}$, tandis qu'en France le taux de ménages immigrés dans ce même type de logements s'élevait à $15 \%$ en $1996^{13}$. Mais les foyers étrangers au Japon ne sont pas répartis de façon homogène : ils se concentrent principalement dans des zones à la périphérie des grandes villes ${ }^{14}$. 
À l'intérieur de ces départements, les foyers étrangers sont particulièrement denses dans quelques cités souvent situées dans les banlieues excentrées à proximité des zones industrielles. Dans une enquête conduite par le ministère du Transport et du Territoire, 29 départements japonais sur 47 affirment qu'il existe dans leur département "des cités à forte population étrangère" (gaikokujin shûjû danchi $)^{15}$. Parmi elles, certaines comptent autant et parfois même plus - de résidents étrangers que japonais : dans la cité A, par exemple, située dans la ville de Toyota, les résidents étrangers, majoritairement des travailleurs brésiliens, représentent plus de $45 \%$ des résidents (4 158 résidents sur 8 568), tandis que dans la cité $B$, située à Chita, à 30 minutes du centre Àl'intérieur de Nagoya en train, ce sont de ces départements, $\quad 60 \%$ des 1850 logements les foyers étrangers sont particulièrement denses dans quelques cités souvent situées dans les banlieues excentrées à proximité des zones industrielles. qui sont habités par des foyers étrangers, principalement des Brésiliens.

Un animateur étudiant travaillant dans une association de capoeira nous raconte son impression lorsqu'il est arrivé pour la première fois dans la cité B : “On aurait dit qu'il n'y avait que des Brésiliens dans cette cité ! Tout ce que j'entendais était portugais. Plein de mecs avec de gros yeux et de belles filles exotiques! Vraiment l'impression d'être au Brésil ${ }^{16}$ !"

La proportion du nombre d'étrangers se manifeste de façon plus spectaculaire encore dans le cadre scolaire : les établissements scolaires et les crèches situés à proximité de ces cités affichent une très forte proportion de ressortissants étrangers. Près de la cité $C$, par exemple, à une heure et demie du centre de Tokyo, où 49,1\% des résidents sont d'origine étrangère - de plus de 20 nationa- lités différentes (Chinois, Vietnamiens, Coréens, Philippins sont les quatre nationalités les plus représentées), à la différence des cités $\mathrm{A}$ et $\mathrm{B}$ où on trouve une grande proportion de Brésiliens -, l'école publique affiche $73,8 \%$, et la crèche $88,1 \%$ d'étrangers sur le nombre total d'enfants ${ }^{17}$. On le comprend aisément, cette situation contribue, parfois de façon radicale, à la transformation structurelle de la société locale, ce que révèlent les propos d'une enseignante travaillant dans cette école publique : "À la fête de l'école, quand je suis arrivée il $y$ a huit ans, les élèves semblaient plus détendus que d'habitude grâce à la présence exceptionnelle de leurs parents, et un élève chinois de CP1 m'a demandé d'un air tout innocent: "Vous êtes quel peuple? - Comment ça, quel peuple? - Oui, de quelle nationalité ? Je suis japonaise. - Oui, mais quoi encore? - Mais je suis japonaise. - C'est tout?' Sa question m'a un peu troublée. Ça fait plus de vingt ans que je suis enseignante et je me suis aperçue que cétait la première fois qu'un élève me posait une question sur ma nationalité. Je n'avais jamais été dans un environnement aussi multiculture ${ }^{18}$."

\section{Les conditions de l'ouverture du parc public aux populations étrangères}

La hausse remarquable des résidents étrangers dans les HLM - leur nombre a plus que quadruplé - ne s'explique pas seulement par l'augmentation de la population étrangère au Japon (on estime qu'elle a doublé en vingt ans ${ }^{19}$ ). Elle est due avant tout au changement de la politique des logements publics, dans un processus qu'on pourrait comparer à celui des HLM en France. Depuis la création de la loi relative aux logements publics 
en 1951, l'État japonais a participé à la construction massive de HLM pour résoudre la pénurie de logements d'après-guerre, en construisant en moyenne 100000 logements par an de la fin des années 1960 au début des années 1970, afin d'accueillir des classes moyennes en pleine croissance. Mais, à partir de 1973, année où le nombre de logements dépasse le nombre de foyers total du pays, la politique des HLM passe de la construction à la gestion. Dans les années 1980, où le niveau de vie s'améliore de façon importante, notamment par les effets de la bulle économique, le problème des logements vacants s'accélère encore, avec le départ des classes moyennes vers le parc privé.

C'est dans ce contexte que le ministère de la Construction $^{20}$ publie, en 1992, une circulaire permettant aux résidents étrangers de bénéficier désormais des mêmes conditions que les Japonais (en fonction d'un examen des dossiers sous critère de revenus) pour habiter dans les HLM. La stratégie institutionnelle de combler les logements vacants en les proposant aux résidents étrangers en augmentation est similaire à celle de la France du milieu des années 1970, après le changement de la politique du logement sous Giscard d'Estaing, ayant pour but de favoriser l'accession à la propriété des classes moyennes ${ }^{21}$. L'entrée des étrangers dans les HLM se fait alors massivement dans des cités qui affichent deux caractéristiques : d'une part, leur localisation désavantageuse (ce sont des cités situées dans des banlieues excentrées et mal desservies, de plus en plus abandonnées par les résidents japonais, un phénomène qui s'accélérera avec la gentrification du centre-ville); d'autre part, la vétusté de leurs bâtiments - beaucoup ont plus de trente-cinq ans d'âge, ce qui est considérable dans un pays sismique où "l'espérance de vie" d'un logement est beaucoup plus courte qu'en Angleterre et aux États-Unis ${ }^{22}$, et où l'ancienneté d'un logement est un élément la plupart du temps négatif pour sa location.

Mais cette concentration provient aussi de la situation spécifique des résidents étrangers. Pour les new comers, il n'est pas toujours facile d'accéder au parc de logements privés. Plusieurs facteurs sont ici en cause et d'abord celui des discriminations xénophobes : selon une enquête réalisée en 1994, $10 \%$ des agences immobilières refusaient catégoriquement toute demande de location de la part des
La stratégie institutionnelle de combler les logements vacants en les proposant aux résidents étrangers en augmentation est similaire à celle de la France du milieu des années 1970 répondaient "en fonction de la catégorie des étrangers", et seulement moins de $20 \%$ acceptaient sans condition de traiter avec des étrangers ${ }^{23}$. De plus, des procédures administratives compliquées empêchent les étrangers d'accéder au parc privé (nécessité d'un garant japonais, exigence d'une compétence linguistique et de connaissances sur la culture japonaise, éléments presque toujours demandés par les propriétaires), alors que dans le parc public, les conditions sont désormais identiques à celles requises pour les Japonais. Une troisième explication relève de la dimension économique : un loyer peu élevé (de 10000 à 30000 yens par foyer pour les HLM, de 30000 à 50000 yens pour les logements sociaux gérés par la Urban Renaissance Agency) et l'absence de caution à verser quand on entre dans un logement public (tandis que dans le parc privé, on doit payer environ trois à six mois de loyer au moment du contrat) favorisent aussi le regroupement des étrangers.

Enfin, la concentration des résidents étrangers s'alimente pour ainsi dire d'elle-même en donnant naissance à un environnement favorable à leur vie quotidienne (développement de commerces 
ethniques, de structures d'entraide et d'information) et où l'on peut vivre en pratiquant sa langue d'origine.

\section{L'opposition entre les résidents japonais et étrangers}

Depuis la fin des années 1990, les problèmes de ces "cités à forte population étrangère" ont commencé à faire la Une des médias. Si certains faits tragiques ont été amplement commentés - comme l'assassinat d'un Brésilien de 14 ans, lynché par un groupe de jeunes Japonais qui "n'aimaient pas les Brésiliens" en $1997^{24}$, ou des oppositions musclées entre les étrangers et l'extrême droite en $1999^{25}$-, de nombreuses situations n'ayant rien de spectaculaire sont vécues et perçues comme des "problèmes", et parfois jugées "très graves" par les résidents.

L'enquête auprès des 47 départements japonais effectuée par le ministère du Territoire nous en donne un aperçu à la fois répétitif et très varié : bruit, non-respect des règles concernant les poubelles, sous-location ou hébergement non autorisé, rassemblement des étrangers dans les parties communes, stationnement illégal, non-scolarisation des enfants, non-participation au comité autogestionnaire des résidents (jichi-kai), non-paiement des frais du comité, difficultés linguistiques ou incompétence en japonais, départ des résidents japonais - tels sont les problèmes évoqués par les gérants des cités HLM ${ }^{26}$.

On voit bien que ces "problèmes" sont presque tous présentés comme étant liés à certains comportements, à certaines attitudes ou certaines pratiques émanant de la population étrangère. On prend ainsi l'habitude de mettre en exergue des problèmes qui seraient récurrents et spécifiques aux résidents étrangers : il s'agit de la "non-intégration", de la "méconnaissance de la culture japonaise" et de la "différence culturelle" des étrangers, trois composantes censées poser problème à la société locale, d'une manière qui rejoint l'analyse des chercheurs qui s'intéressaient dès le début des années 1990 aux oppositions problématiques entre résidents japonais et étrangers, comme Kurumi Tsuzuki ${ }^{27}$. À partir d'un tel constat, il est logique que les solutions proposées s'inscrivent dans une perspective culturelle. C'est ainsi que se sont développées, promues par la ville ainsi que par des associations ou les comités d'autogestion des résidents eux-mêmes, de nombreuses initiatives de soutien aux étrangers, mais qui proposent des aides uniquement en matière de langue (cours de japonais, service d'interprétariat et de traduction), d'éducation (soutien scolaire, ateliers pour expliquer aux adultes comment vivre dans les cités japonaises, permanence juridique pour les résidents étrangers) et de culture (organisation de fêtes et d'échanges culturels).

Une question se pose cependant, qui pointe peutêtre les limites de cette politique et du raisonnement théorique peu convaincant qui la sous-tend : s'il y a des problèmes dans les cités à forte population étrangère, ceux-ci sont-ils vraiment uniquement dus aux étrangers ? Autrement dit : la concentration d'étrangers dans une cité génère-telle systématiquement des problèmes? Cette question peut sembler très simple, mais de la réponse qu'on lui donne dépend toute la configuration de la problématique qui nous occupe, aussi bien dans sa formulation théorique que dans ses conséquences pratiques et politiques.

Ainsi, certains travaux montrent de façon empirique que la cohabitation entre résidents japonais et étrangers ne provoque pas partout, toujours et systématiquement, des conflits ; au contraire, elle peut même très bien fonctionner ${ }^{28}$. Pour ne citer qu'un exemple, lors de notre enquête dans une cité à Iwata

24. Pour plus de détails, voir Rumiko Nishino, Naze Herculano wa Korosaretanoka? (Pourquoi Herculano a-t-il été assassiné? Le lynchage d'un garçon brésilien d'origine japonaise), Tokyo, Akashi-Shoten, 1999. 25. Voir "Tensions dans la cité Homi”, in Chûnichi Shimbun, 8 juin 1999. 26. Source : ministère du Transport et du Territoire, 2007 (http://www.mlit.go.jp/kokudokeikaku/souhatu/h18seika/o4kitakantou/O4_03hon pen2.pdf). 27. Kurumi Tsuzuki, “Chihôsangyôtoshi ni okeru Burazilujin no ukeireto chii no henyô" ("L'installation des Brésiliens et la transformation de la société locale. Des conflits et de la tension au vivre ensemble"), in Toshikagaku URC, 1996. 28. Voir aussi Ashita Matsumiya, "Sorewa mondai dewanai kamoshirenai" (“Ce n'est sans doute pas un problème : à propos d'une cité à forte population étrangère"), in Alta, décembre 2008, ou Yoshiko Inaba, “Étude sur la réalité de l'installation des étrangers dans les HLM", art. cit, 2008 
Doyas de Kotobuki. "Doya” est un terme couramment utilisé pour désigner des logements sommaires des travailleurs journaliers aujourd'hui âgés, sans familles ni retraites.

(c) CAmille Millerand

(département de Shizuoka), les habitants affirment qu'il y a "très peu de problèmes" entre les Japonais et les étrangers, bien que le taux de ces derniers avoisine les $50 \%{ }^{29}$. Partant de ces résultats, il est donc plus judicieux de supposer que ce n'est pas nécessairement la cohabitation qui pose problème, mais la cohabitation dans certaines conditions. Pour comprendre lesquelles, il est essentiel de se pencher très précisément sur les caractéristiques des cités où les problèmes se posent.

\section{L'impact du vieillissement de la population des cités}

Notre enquête nous permet de constater que parmi les cités à forte population étrangère, celles où il y a un malaise ou des conflits entre résidents japonais et étrangers se distinguent non pas par les caracté- ristiques des résidents étrangers mais par celles... des résidents japonais. Et plus précisément par les caractéristiques suivantes : la proportion importante des personnes âgées (dans ces trois cités, tous les nouveaux résidents japonais depuis cinq ans ont 70 ans ou plus), leur situation d'isolement familial (plus de la moitié d'entre eux vivent seuls) et leur condition socio-économique fragile (un tiers d'entre eux vivent grâce au "revenu d'assistance minimal", le seikatsu-hogo, équivalent du RSA en France). Or il convient de souligner qu'une telle concentration de personnes âgées souvent isolées et fragiles dans les cités s'est accélérée en 1996 à la suite de la réforme de la loi relative aux conditions d'accès aux HLM. Dans le double objectif d'une meilleure gestion du stock et de la distribution de logements aux personnes en grande difficulté, l'État a baissé le revenu maximum des résidents de $20 \%$, sauf pour les personnes ayant plus de 65 ans $^{30}$, en acceptant, de surcroît, des personnes seules de plus de 50 ans (avant, seuls les couples avec ou sans enfants pouvaient y 
accéder). Ces changements prouvent la volonté de l'État de transformer les HLM, du moins en partie, en des sortes d'hospices, en y favorisant l'implantation - ou devrait-on dire le refoulement ? - des personnes âgées et en provoquant ainsi le départ des classes moyennes et des couches les plus jeunes de la population qui ne peuvent plus y rester, entre autres à cause de l'augmentation des loyers ${ }^{31}$.

Ce constat nous amène donc à considérer les problèmes des oppositions entre résidents étrangers et japonais dans les cités à forte population étrangère sous un angle neuf, et avec des instruments de mesure et d'analyse qui devraient être différents. On constate, en effet, que les oppositions en question ne se produisent pas nécessairement entre les Japonais et les étrangers, comme on le souligne trop souvent, de manière aussi forte que rapide, tant dans les médias que dans certaines recherches académiques, mais qu'il s'agit bien souvent en fait de problèmes générationnels (parmi les résidents étrangers de la cité C, $22 \%$ ont moins de 20 ans, $40 \%$ entre 20 et 30 ans, tandis que les résidents japonais ont majoritairement plus de 65 ans), de décalages dans les configurations familiales (les foyers étrangers ont souvent des enfants en bas âge, alors que les personnes âgées japonaises vivent souvent seules aujourd'hui), ou encore de problèmes de style de vie qui ne sont pas obligatoirement - ni même majoritairement - réductibles à des perspectives culturelles. Certains sont tout simplement liés à des contraintes professionnelles ou à des différences d'emploi du temps (ainsi, les étrangers travaillent parfois jusqu'à des heures tardives et rentrent tard, invitent des amis le soir, alors que les Japonais âgés se couchent bien plus tôt).

La communauté des cités HLM n'est donc pas nécessairement traversée par le seul clivage entre Japonais et étrangers, mais aussi par bien d'autres variables. Cependant, ces résidents, malgré la grande hétérogénéité qui les caractérise - étrangers de diverses nationalités et population japonaise, jeunes et personnes âgées, vivant seuls ou en famille, travailleurs ou retraités, etc. -, partagent un point commun qui devrait inciter à relativiser l'opposition que, fort schématiquement, on cherche à dessiner entre eux. Quelle que soit la catégorie à laquelle ils appartiennent, ils se trouvent souvent dans une situation précaire et fragile. Les résidents japonais ont souvent peu de revenus et ne peuvent pas compter sur des soutiens familiaux, tandis que les résidents étrangers occupent des emplois très instables. Cette précarité s'est notamment révélée au grand jour après la crise économique de 2008, qui a laissé beaucoup de travailleurs étrangers au chômage. Une résidente d'origine brésilienne de la cité A nous parle ainsi de l'atmosphère de crise, peut-être encore plus spectaculaire ici qu'ailleurs, à cause de la baisse d'activités chez Toyota, principal fournisseur d'emplois de la région, qui a enregistré une baisse de son chiffre d'affaires pour la première fois depuis sa fondation. Ses paroles sont révélatrices: "Depuis un mois, on héberge la famille de mon neveu chez nous : cinq personnes... mais c'est partout comme ça... On dirait que c'est le quartier entier qui a fait faillite 32 !"

Autrefois lieu composé des classes modestes et moyennes, les cités HLM japonaises se sont aujourd'hui transformées en des lieux où se regroupent des résidents socio-économiquement précaires, qu'ils le veuillent ou non.

\section{Instabilité et insécurité}

Face à la fragilisation des situations et des liens sociaux, les comités d'autogestion des résidents (jichi-kai) et plusieurs associations de soutien prennent diverses initiatives à l'intérieur des cités pour renforcer le réseau local de solidarité : ici, on constitue un conseil des résidents étrangers ; là, on crée un lieu d'échanges entre les résidents japonais et étrangers. Dans une autre cité encore, on met en place un soutien éducatif et juridique pour les habitants étrangers ou des panneaux d'informations multilingues, etc. Si la cohabitation des différents résidents fonctionne bien dans certaines cités, 
c'est largement grâce à ces initiatives qui viennent d'“en bas", vu le manque rédhibitoire de politiques publiques en ce domaine.

Mais une nouvelle réforme de la loi HLM, en novembre 2005, a rendu encore plus difficile le maintien de la "communauté" des résidents. Celleci consiste en deux orientations distinctes. La première se traduit par le prolongement de la réforme de 1996 pour remplacer les classes moyennes avec un revenu plus ou moins stable par des personnes en situation économique plus précaire : les classes moyennes doivent maintenant payer un loyer aussi élevé que s'ils étaient dans le parc privé, elles n'ont donc plus aucun intérêt de continuer à habiter dans une HLM, tandis que les personnes âgées, mais aussi des personnes appartenant à d'autres catégories perçues comme "fragiles", à savoir les handicapés mentaux et physiques ainsi que les femmes victimes de violences domestiques, sont désormais autorisées à entrer dans une HLM, même quand elles sont célibataires (avant, une personne seule ne pouvait pas y habiter). Cet ensemble de mesures renforce donc le caractère "social" des HLM. Dans la cité $C$, située dans la banlieue de Tokyo, le président du comité d'autogestion témoigne: "Ils [les personnels de la municipalité] ne nous envoient que des personnes en grande difficulté, mais nous ne sommes pas des centres sociaux, ni des travailleurs sociaux! Ils font partir les jeunes Japonais qui ont pu soutenir les activités du comité d'autogestion, alors que nous avons besoin d'eux... Nous ne pouvons plus maintenir une vie de communautée 33 ."

La deuxième orientation de la réforme de 2005 va exactement dans la même direction : elle consiste en une "flexibilisation" des conditions d'accès des résidents, qui se manifeste de deux manières. D'un part, le durcissement des critères pour la succession d'un logement HLM : désormais, l'enfant d'un locataire de HLM n'a plus le droit d'y habiter après le décès du parent qui le logeait. D'autre part, les nouvelles formes d'offres locatives pour une durée déterminée : les familles ayant au moins un enfant de moins de 6 ans peuvent entrer dans une HLM, mais seulement pour une durée maximale de neuf ans, tandis que les sans domicile fixe ne peuvent louer un logement HLM que pour une durée de deux ans.

Notre enquête montre donc que cette réforme pousse encore la transformation des HLM en ce qu'il faut bien se résoudre à appeler des "centres d'hébergement social”, où se regroupent les pauvres ainsi que les personnes âgées et handicapées en situation instable, ou encore les foyers monoparentaux sans père, qui sont dans une grande précarité ${ }^{34}$. Tous ces facteurs contribuent à renforcer l'image négative des HLM. Pour les gens vivant

à l'extérieur des cités, cellesci sont de plus en plus perçues comme des "zones de Les effets de stigmatisation spatiale, qui conduisent à affaiblir la solidarité entre résidents et à accroitre mauvaise réputation". Pour les gens de l'intérieur, si certains disent n'avoir jamais vécu de discriminationscausées par leur lieu d'habitat, le sentiment d'hostilité, renforcent donc les difficultés de cohabitation équilibrée. d'autres ressentent un sentiment très négatif qui les pousse à cacher leur adresse stigmatisée. Dans un double mouvement de spirale et d'enfermement, ils se résignent aussi à ne pas pouvoir inviter des gens chez eux.

\section{Les logiques de clivage}

Pour atténuer ce sentiment d'humiliation, certains résidents empruntent des stratégies de "distinction" en s'écartant et en se différenciant des autres résidents (la non-participation aux activités organisées par le comité d'autogestion des résidents en est un exemple typique), selon un procédé déjà analysé par Loïc Wacquant dans sa recherche sur les banlieues françaises ${ }^{35}$. Alors se développent des phénomènes de désolidarisation, de délitement et de fragmentation qui brisent les mythes de la banlieue homogène et del'harmonie sociale surlesquels s'était construite la société japonaise. 
Du côté des résidents japonais, les plaintes à propos des comportements de leurs voisins étrangers sont nombreuses et récurrentes: "Ils ont acheté une télévision dernier cri alors qu'ils reçoivent le RMI", "Ma voisine touche des aides sociales, mais elle travaille dans un bar à hôtesses!", "Ils n’ont pas déclaré leurs enfants auprès de la mairie", "ils sont en sous-location", "C'est un mariage blanc", "Il y a des divorces blancs", "Elles touchent des allocations pour mères célibataires pour les envoyer à leurs familles restées au pays !'... Certains résidents, souvent âgés, "traumatisés" par le bruit que font les résidents étrangers, entendent même des "bruits qui n'existent pas", devenant presque paranoïaques : un résident japonais de 72 ans est ainsi convaincu que "leurs enfants font exploser des pétards jusque dans [s]a maison ${ }^{36 "}$.

Mais, du côté des résidents étrangers, on observe un mécontentement parfois très prononcé. Certains témoignent de leur crainte d'être mal vus par d'autres résidents étrangers, car "trop proches des Japonais", tandis que d'autres sont mécontents des résidents japonais qui "ne cessent de [les] engueuler, alors qu'[ils] paient autant de loyer et d'impôts qu'eux". D'autres espèrent qu'il n'y aura pas plus de résidents étrangers comme eux dans leur cité, craignant un renforcement des effets de "stigmatisation spatiale" dont ils sont déjà l'objet.

D'autres, enfin, manifestent leur souhait de ne pas être confondus avec leurs compatriotes, qui viennent certes du même pays mais n'appartiennent pas à la même classe sociale ou ne sont pas aussi intégrés qu'eux, comme le montrent les propos d'un résident brésilien de la cité A : "On nous parle des problèmes de bruit, des poubelles, mais tout le monde respecte les règles, sauf une petite dizaine qui foutent le bazar, et tout de suite les 3000 autres Brésiliens sont condamnés!"

Les effets de stigmatisation spatiale, qui conduisent à affaiblir la solidarité entre résidents et à accroître le sentiment d'hostilité, renforcent donc les difficultés de cohabitation équilibrée. Ces quartiers stigmatisés provoquent le départ de nombreuses familles japonaises (la population japonaise a diminué en moyenne de $25 \%$ en cinq ans dans les trois cités de notre enquête) et étrangères les moins démunies. Les familles ayant un revenu stable, les personnes réussissant dans leurs études et susceptibles de devenir "leaders des résidents étrangers" ont aussi tendance à quitter la cité. Il ne reste alors que la population "qui ne peut pas faire autrement" : étrangers en situation économique précaire ou Japonais âgés avec peu de ressources, souvent seuls ou avec des handicaps ${ }^{37}$, abandonnés à eux-mêmes, dans une tension chaque jour renouvelée et exacerbée. Cette tension prend parfois des formes radicales, notamment lorsqu'elle est confrontée à une certaine récupération politique. Déjà, à la fin des années 1990, une frange de l'extrême droite japonaise était venue provoquer les résidents brésiliens d'une cité de la banlieue de Nagoya, entraînant par la suite des conflits violents, dans une affaire relayée à plusieurs reprises par les médias ${ }^{38}$. Mais, plus récemment, les membres du groupement de la "nouvelle extrême droite", connus sous le nom de Zaitoku-kai (Collectif des citoyens contre les privilèges des résidents étrangers au Japon), exploitent systématiquement ce terrain : depuis 2009, ils multiplient les actions et les manifestations dans des cités à forte population étrangère, en diffusant des discours ouvertement xénophobes et racistes, ce qui inquiète de plus en plus les habitants ${ }^{39}$.

\section{La banlieue japonaise au miroir de la banlieue française}

Les problèmes qui se posent aujourd'hui dans les banlieues japonaises sont souvent vus uniquement sous l'angle d'une opposition entre Japonais et étrangers, ou de "problèmes liés à l'augmentation des résidents étrangers". On l'a vu, cette présentation si courante ne correspond pas à la réalité du terrain. Elle ne résiste ni à un examen attentif de 
la situation, ni à une analyse plus fine et plus poussée. En réalité, les "oppositions" qui se développent effectivement dans les banlieues japonaises sont aussi - et peut-être avant tout - le résultat de politiques qui concentrent de plus en plus de personnes fragiles dans un espace de plus en plus stigmatisé. Elles résultent d'un malaise, d'un sentiment d'insécurité sociale grandissant, et se nourrissent des transformations sociales entraînées par la globalisation économique - c'est ce que nous avons tâché de souligner dans le présent article.

Pour faire face à ce malaise, qui se traduit en partie aujourd'hui par la montée du nationalisme et de la xénophobie, il n'est pas suffisant d'intervenir en matière linguistique ou culturelle, sous la houlette des beaux sentiments ou du slogan galvaudé du "vivre ensemble". Il est également indispensable de trouver des solutions à l'augmentation des inégalités et à la précarisation socio-économique dont souffrent les résidents des parcs publics : c'est bien celles-ci qui constituent à mes yeux le terreau de l'insécurité sociale de plus en plus diffuse qu'on peut constater chaque jour, et qui se traduit par un sentiment d'insécurité physique lié à la présence des étrangers, comme le constate Robert Castel au sujet des liens incontestables entre sentiment d'insécurité et instabilité sociale ${ }^{40}$.

Loin de l'image de l'homogénéité qui continue à nourrir le mythe de la société japonaise, certaines banlieues japonaises constituent donc aujourd'hui un espace hétérogène et instable, où cohabitent des populations d'origines variées et de statuts différents, dans une sorte de théâtre local où se font sentir les effets les moins glorieux de la globalisation économique. Mutatis mutandis, leur situation peut dans certaines limites - mais à bien des égards - faire penser à la situation française : si la situation japonaise peut paraître beaucoup moins "grave" que celle de la France, s'il n'y a pas autant de massification (les cités à problèmes y sont à la fois moins nombreuses et localisées dans quelques lieux précis), ni d'intensification du phénomène (on n'a pas encore assisté à des émeutes comme on en voit de manière récurrente en France), leur avenir est pourtant loin d'être rose. Les problèmes principaux résident dans le manque de politique japonaise cohérente et de vision à long terme en la matière.

On peut craindre que le fait qu'une bonne partie des enfants de ces étrangers se trouvent en état de non-scolarisation - ou de semi-scolarisation - ne soit que le prélude à une
Les "oppositions" qui se développent effectivement dans les banlieues japonaises sont aussi - et peut-être avant tout - le résultat de politiques qui concentrent de plus en plus de personnes fragiles dans un espace de plus en plus stigmatisé. tion $^{41}$. Il convient donc d'insister pour finir sur un point : si ces banlieues ne sont pas si nombreuses aujourd'hui, elles ne constituent pas pour autant des cas isolés, ni le reliquat inévitable d'un progrès économique dont on nous chante par ailleurs les louanges, au prix d'un décalage de plus en plus criant avec la réalité. Elles me semblent au contraire un laboratoire pour les problèmes qui se posent dès maintenant et ne manqueront pas de se poser demain dans nombre de zones urbaines, y compris au Japon, qu'on le veuille ou non.

Malgré le caractère mondial du phénomène qui nous préoccupe ici, la tendance générale semble être de continuer à considérer les problèmes dans un cadre national, en considérant qu'il s'agit de problèmes spécifiques, "bien de chez nous" pour ainsi dire. Il est pourtant impératif, à notre époque de mondialisation, de ne pas sombrer dans une myopie analytique réduisant l'observation et l'interprétation des situations à une explication par des particularismes nationaux. C'est pourquoi une perspective comparatiste plus large, examinant les effets de miroir, de symétrie ou de contraste entre l'Europe, l'Amérique du Nord et le Japon, me semble aujourd'hui si importante. 